\title{
Enzyme Activities Related to Fatty Acid Synthesis in Developing Mammalian Lung
}

\author{
Ian Gross and Joseph B. Warshaw ${ }^{[34]}$ \\ Division of Perinatal Medicine, Departments of Pediatrics and Obstetrics and Gynecology, Yale University School of Medicine, \\ New Haven, Connecticut, USA
}

\section{Extract}

Fatty acids are important components of pulmonary surface-active lipids and lung membranes. We have investigated fatty acid synthesis in developing rabbit lung by measuring the rate of incorporation of $\left({ }^{14} \mathrm{C}\right)$ acetyl or $\left({ }^{14} \mathrm{C}\right)$ malonyl-CoA into pentaneextractable fatty acids. Because the lung can incorporate exogenously supplied as well as endogenously synthesized fatty acid into phospholipid, parallel studies of hepatic fatty acid synthesis were carried out.

Fatty acids can be synthesized in fetal rabbit lung by the de novo pathway and to a lesser extent by chain elongation. The activity of enzymes related to de novo fatty acid synthesis is at adult levels by 23 days of fetal life and remains fairly constant thereafter. Enzymes associated with hepatic de novo fatty acid synthesis reach very high levels of activity during fetal life but this decreases by $50 \%$ after birth, in association with the onset of suckling. Activity of the enzymes of de novo pulmonary synthesis appears to be unaffected by birth and onset of nutritional intake.

Pulmonary and hepatic fatty acid elongation do not appear to be major routes of fatty acid synthesis in the fetal rabbit. Microsomal elongation activity is low in the fetus and on the first day of life, adult activity being fourfold greater. Pulmonary mitochondrial elongation becomes progressively more active during fetal life, but quantitatively appears less important than de novo synthesis.

Gas-liquid chromatography of the fatty acids synthesized by fatty acid synthetase in the supernatant fraction of lung homogenates revealed that the main product was palmitic acid, a major component of dipalmityl lecithin which is an important surfaceactive lipid. The maior products of pulmonary mitochondrial and microsomal fatty acid elongation were stearic acid and 20- and 22-carbon unsaturated fatty acids.

The early maturation of the enzymes of the de novo pathway ensures a source of fatty acid for lung membrane growth and for surfactant formation when the pathways for lecithin synthesis mature.

\section{Speculation}

Inasmuch as the enzymes associated with de novo fatty acid synthesis are active before the pathway for lecithin synthesis matures, fatty acid synthesis is probably not the ratelimiting factor in surfactant production in the rabbit fetus. The role of other lecithin precursors such as choline remains to be investigated.

Early dietary intake does not appear to influence pulmonary fatty acid synthesis, but may play an important role in overall pulmonary lipid metabolism and the regulation of lung surface activity. 


\section{Introduction}

The surface activity of the alveolar lining layer is believed to reside primarily in a phospholipid fraction secreted into the alveolus. Dipalmityl lecithin is thought to be an important compound which confers this activity [14]. Although much work has been done on the biosynthesis of lecithin in pulmonary tissue during development [7-9], little is known of the capacity of developing lung to synthesize lecithin precursors, particularly pulmonary fatty acids.

Initial investigations of pulmonary fatty acid synthesis have produced contradictory reports. Tombropolous [19] reported that long chain fatty acid synthesis in the rat lung was most active in a mitochondrial-rich fraction. De novo fatty acid synthesis in the supernatant fraction of the cell and microsomal fatty acid elongation were relatively inactive. Chida and Adams [4], who used fetal lamb lung slices, showed that the fetal lung could synthesize palmitic and other long chain fatty acids. They did not, however, determine whether synthesis was de novo or by chain elongation, nor did they investigate developmental profiles in any detail. Schiller and Bensch [17] demonstrated subsequently that in addition to mitochondrial and microsomal fatty acid elongation, de novo synthesis by the supernatant system was active in adult rabbit lung.

Because of the importance of fatty acids as lecithin precursors, we have investigated the activity of the enzymes related to de novo synthesis of long chain fatty acids and mitochondrial and microsomal fatty acid elongation by subcellular fractions of fetal rabbit lung. The lung can incorporate exogenously supplied as well as endogenously synthesized fatty acid into phospholipids [10]. We have therefore carried out parallel studies of fatty acid synthesis by the liver.

\section{Materials and Methods}

Timed pregnant rabbits were obtained from Camm Research Institute [24]. Fetuses from 23 to 30 days of gestational age were removed from the mother by caesarian section and killed immediately by decapitation. Newborn rabbits were allowed to feed from the mother for 1 day and were then killed by decapitation. Lungs and livers from 8-16 fetuses or 3-4 postnatal animals were used for each experiment. The lungs and liver were excised immediately and chilled at $4^{\circ}$ in $0.25 \mathrm{M}$ sucrose, $10 \mathrm{~mm}$ potassium phosphate, $\mathrm{pH} 7.4$, and $1.0 \mathrm{~mm}$ EDTA (PSE). After trimming of connective tissue and large vessels, the organs were finely minced. Several washes with PSE were carried out to remove blood and the minced tissue was then suspended in 8 volumes of PSE. The tissue was homogenized using a Brinkmann P-20 homogenizer [25] for $20 \mathrm{~s}$ at a rheostat setting of 2 .

The homogenate was centrifuged at $1,000 \times g$ for 10 min to remove nuclei and debris. A mitochondrial fraction was isolated by centrifugation at $10,000 \times g$ for $10 \mathrm{~min}$; the pellet was washed twice at $15,000 \times \mathrm{g}$ for $10 \mathrm{~min}$ and finally suspended in $0.25 \mathrm{~m}$ sucrose, 10 mm Tris-Cl, pH 7.4.

A microsomal fraction was isolated by centrifugation of the $10,000 \times g$ supernatant at $150,000 \times g$ for $1 \mathrm{hr}$. The resultant pellet was suspended in PSE. The $150,000 \times g$ supernatant was retained for study of $d e$ novo fatty acid synthesis. Subcellular fractionation of liver homogenates was carried out in the same way except that mitochondria were separated at $8,000 \times g$. All procedures were performed at $4^{\circ}$ and the various fractions stored at $-70^{\circ}$ for later assay.

Protein determination was done by a modification of the biuret method [13]. Mitochondrial activity was measured polarographically by measuring cytochrome oxidase activity [20]. Over $75 \%$ of recoverable cytochrome oxidase was found in the $10,000 \times g$ pellet.

Assays

Fatty acid synthesis was assayed by measuring the rate of incorporation of $\left({ }^{14} \mathrm{C}\right)$ acetyl-CoA or $\left({ }^{14} \mathrm{C}\right) \mathrm{malo}$ nyl-CoA into pentane-extractable fatty acids. Saturation levels of all substrates were used.

Fatty acid synthetase. The assay included malonylCoA, $150 \mathrm{nmol}$ which contained $\left(1,3-{ }^{14} \mathrm{C}\right)$ malonyl-CoA (sp act $27 \mathrm{mCi} / \mathrm{mm}$ ), 40,000 cpm; acetyl-CoA, $50 \mathrm{nmol}$; potassium phosphate buffer, pH 6.8, $100 \mu$ mol; ATP, 4 $\mu \mathrm{mol}$; NADP, $1 \mu \mathrm{mol}$; NADH $1 \mu \mathrm{mol}$; $0.75 \mathrm{mg}$ supernatant protein, and water to a final volume of $1 \mathrm{ml}$.

Acetyl-CoA carboxylase (EC. 6.4.1.2). This was assayed indirectly by determining the rates of incorporation of $\left({ }^{14} \mathrm{C}\right)$ acetyl-CoA into long chain fatty acids. As we were primarily interested in determining developmental profiles, this assay was chosen as it allows determination of the activity of crude supernatant fractions and avoids day-to-day variation in purification techniques. An excess of fatty acid synthetase in the reaction mixture was demonstrated and shown not to be rate limiting in the reaction. The assay contained acetyl-CoA, $100 \mathrm{nmol}$ with $\left(1-{ }^{14} \mathrm{C}\right)$ acetyl-CoA (sp act 15 $\mathrm{mGi} / \mathrm{mm}), 100,000 \mathrm{cpm}$; potassium phosphate, $\mathrm{pH} 6.3$, $100 \mu \mathrm{mol}$; sodium isocitrate, $20 \mu \mathrm{mol} ; \mathrm{MnCl}_{2}, 7 \mu \mathrm{mol}$; $\mathrm{KHCO}_{3}, 60 \mu \mathrm{mol}$; EDTA, $3 \mu \mathrm{mol}$; bovine serum albumin, $2 \mathrm{mg}$; NADPH, $1.5 \mu \mathrm{mol}$; ATP, $10 \mu \mathrm{mol} ; 1 \mathrm{mg}$ 
supernatant protein, and water to a final volume of 1 $\mathrm{ml}$. The final $\mathrm{pH}$ after addition of bicarbonate was 6.8 .

Microsomal fatty acid elongation. The assay contained malonyl-CoA, 100 nmol with $\left(1,3-{ }^{14} \mathrm{C}\right)$ malonylCoA (sp act $27 \mathrm{mCi} / \mathrm{mm}$ ), 100,000 cpm; Tris-Cl, pH 7.5, $50 \mu \mathrm{mol} ; \mathrm{KCl}, 90 \mu \mathrm{mol}$; EDTA, $1 \mu \mathrm{mol} ; \mathrm{MgCl}_{2}, 5 \mu \mathrm{mol}$; $\mathrm{KCN}, 5 \mu \mathrm{mol}$; palmityl-CoA, $15 \mathrm{nmol}$; NADH, $5 \mu \mathrm{mol}$; ATP, $10 \mu \mathrm{mol}$; microsomal protein, $0.75 \mathrm{mg}$, and water to a final volume of $1 \mathrm{ml}$.

Mitochondrial fatty acid elongation. The assay contained acetyl-CoA, $100 \mu \mathrm{mol}$, with $\left(1-{ }^{14} \mathrm{C}\right)$ acetyl-CoA (sp act $15 \mathrm{mCi} / \mathrm{mM}$ ), $100,000 \mathrm{cpm}$; potassium phosphate, $\mathrm{pH} 7.0,50 \mu \mathrm{mol}$; palmitylcarnitine, $15 \mathrm{nmol}$; antimycin A, $3 \mu \mathrm{g}$; NADH, $3 \mu \mathrm{mol}$; NADPH, $2 \mu \mathrm{mol}$; ATP, $10 \mu \mathrm{mol}$; mitochondrial protein $0.75 \mathrm{mg}$, and water to a final volume of $1 \mathrm{ml}$.

\section{Assay Conditions}

Each determination was done in triplicate. The reactions were incubated for $1 \mathrm{hr}$ at $37^{\circ}$ with occasional agitation. Assays for mitochondrial and microsomal fatty acid synthesis were carried out under a constant stream of nitrogen. The reactions were terminated by the addition of $1 \mathrm{ml}$ of $30 \%(\mathrm{w} / \mathrm{v})$ potassium hydroxide and the fatty acids saponified by heating at $70^{\circ}$ for $30 \mathrm{~min}$. After acidification with $1.2 \mathrm{ml} 5 \mathrm{~N}$ hydrochloric acid, free fatty acids were extracted twice with 5 volumes pentane and transferred to scintillation vials. The pentane was evaporated under nitrogen and after addition of a 2,5-diphenyloxazole and toluene fluor, radioactivity was measured in a Beckman scintillation spectrometer [26].

\section{Product Identification}

Decarboxylation of pentane extracted fatty acids was done by the method of Brady et al. [2]. For gas-liquid chromatography, fatty acids were methylated according to the method of Stoffel et al. [18]. Separation was carried out using a Varian model 2100 gas liquid chromatograph [27] equipped with a no. 02-00142-00 microsample splitter-collector and glass columns (6 foot by $2 \mathrm{~mm}$ ) packed with $15 \%$ Apiezone $\mathrm{L}$ on Gas-Chrom $\mathrm{P} / 100-200$ mesh. Temperature was $200^{\circ}$.

$\left(1-{ }^{14} \mathrm{C}\right)$ Acetyl-CoA and $\left(1,3{ }^{14} \mathrm{C}\right)$ malonyl-CoA were obtained from New England Nuclear [28]. Acetyl-CoA, palmityl-CoA, and malonyl-CoA were obtained from Sigma Chemical Company [29]. Palmitylcarnitine was obtained from Otsuka Pharmaceutical Company [30].

\section{Results}

Assays for the various pathways were optimized using adult rabbit lung. All activities were linear with increasing protein. $\mathrm{pH}$ variation between 6.5 and $7.0 \mathrm{had}$ little effect on fatty acid synthetase activity and mitochondrial fatty acid elongation, but the activity of acetyl-CoA carboxylase in both lung and liver dimin. ished when the buffer $\mathrm{pH}$ was above 6.8 .

\section{Developmental Profiles}

As shown in Figure 1, fatty acid synthetase activity of fetal lung was constant from 23 days of fetal development. A small increase in activity occurred 1 day before birth. The values obtained during fetal life were approximately the same as in adult control subjects. In contrast, hepatic fatty acid synthetase activity was extremely high during fetal development but decreased by $50 \%$ immediately after birth in association with the onset of suckling.

Fatty acid synthesis from acetyl-CoA which we have utilized as an indirect measure of acetyl-CoA carboxylase activity (Fig. 2) followed a pattern similar to that of fatty acid synthetase. Pulmonary activity was again fairly constant during fetal life and also showed a small peak 1 day before birth. Hepatic acetyl-CoA carboxylase activity was high in the fetus and similar to hepatic fatty acid synthetase, fell markedly after birth.

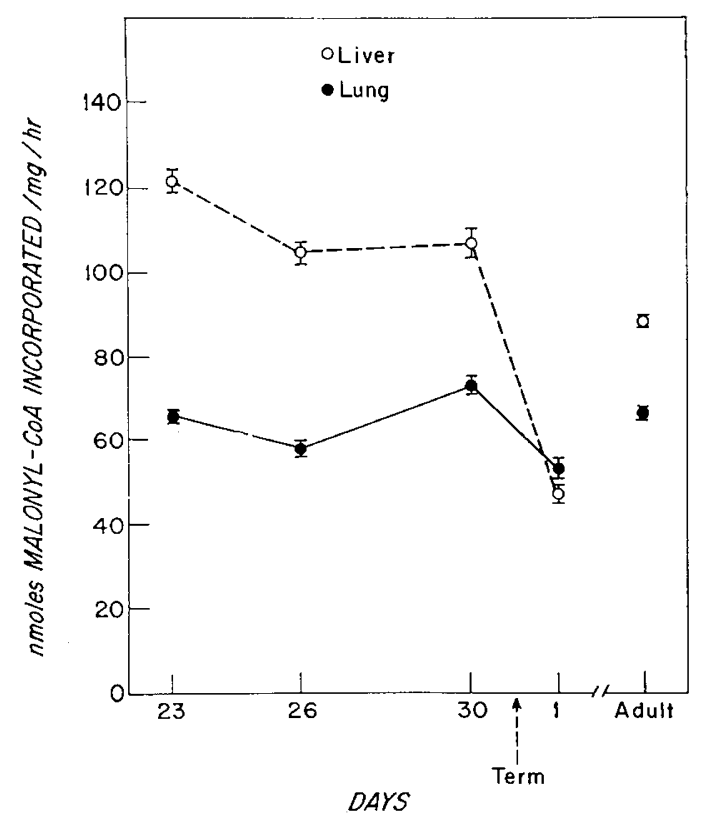

Fig. 1. Developmental profile of pulmonary and hepatic fatty acid synthetase. Assay conditions are as described in the text. Vertical lines represent SEM of three to six experiments. 


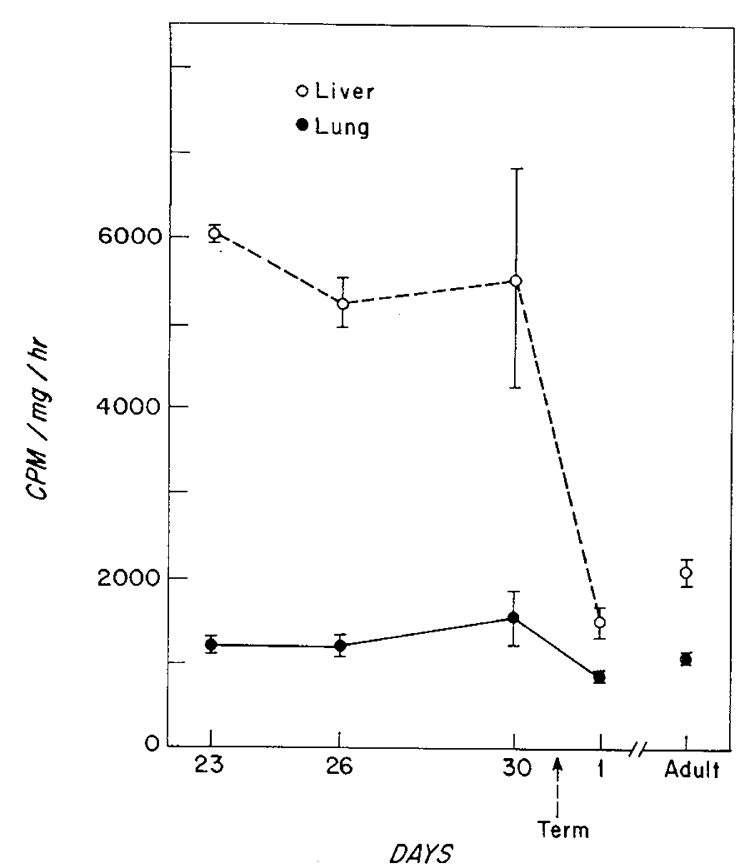

Fig. 2. Developmental profile of pulmonary and hepatic acetylCoA carboxylase. Assay conditions are as described in the text.

As the carboxylase assay utilized was an indirect one, activity is expressed as counts per minute per milligram per hour.

Microsomal fatty acid elongation (Fig. 3) in both lung and liver was relatively inactive in the fetal rabbit. Both activities were $20-25 \%$ of adult control sub-

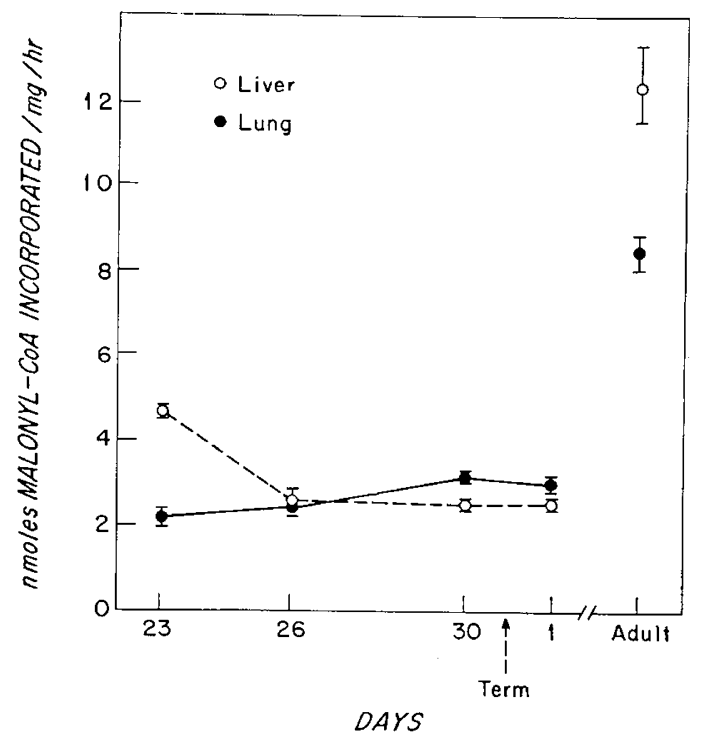

Fig. 3. Developmental profile of pulmonary and hepatic microsomal fatty acid elongation. Assay conditions are as described in the text. jects. Unlike de novo synthesis, there was no immediate change in activity after birth.

As is shown in Figure 4, mitochondrial fatty acid elongation activity increased gradually during fetal lung development. Values approximating those in the adult were attained by 30 days of gestational age. There was no change in activity associated with birth. Elongation activity of fetal liver was similar to fetal lung but was only $50 \%$ that of adult controls.

\section{Product Identification}

Fatty acid decarboxylation was undertaken to discriminate between de novo synthesis and fatty acid chain elongation. In this reaction the carboxyl carbon of the fatty acid is released as $\mathrm{CO}_{2}$. If palmitic acid is the end product of the fatty acid synthetase system, 7 labeled carbon atoms derived from $\left({ }^{14} \mathrm{C}\right)$ malonyl-CoA should be incorporated into each molecule of palmitic acid $\left[\mathrm{CH}_{3} \cdot \mathrm{CH}_{2}\left({ }^{14} \mathrm{CH}_{2} \cdot \mathrm{CH}_{2}\right)_{6} \cdot \mathrm{CH}_{2} \cdot{ }^{14} \mathrm{COOH}\right]$ and $1 / 7$ or $14.3 \%$ of the radioactivity should be recoverable from the carboxyl carbon. In one stage elongation, 1 labeled carbon atom from acetyl or malonyl-CoA will be incorporated into the carboxyl end of the fatty acid molecule $\left[\mathrm{CH}_{3} \cdot\left(\mathrm{CH}_{2}\right)_{\mathrm{n}} \mathrm{CH}_{2} \cdot{ }^{14} \mathrm{COOH}\right]$. Thus all of the radioactivity should be recoverable from the carboxyl carbon. If more than one stage elongation occurs, the

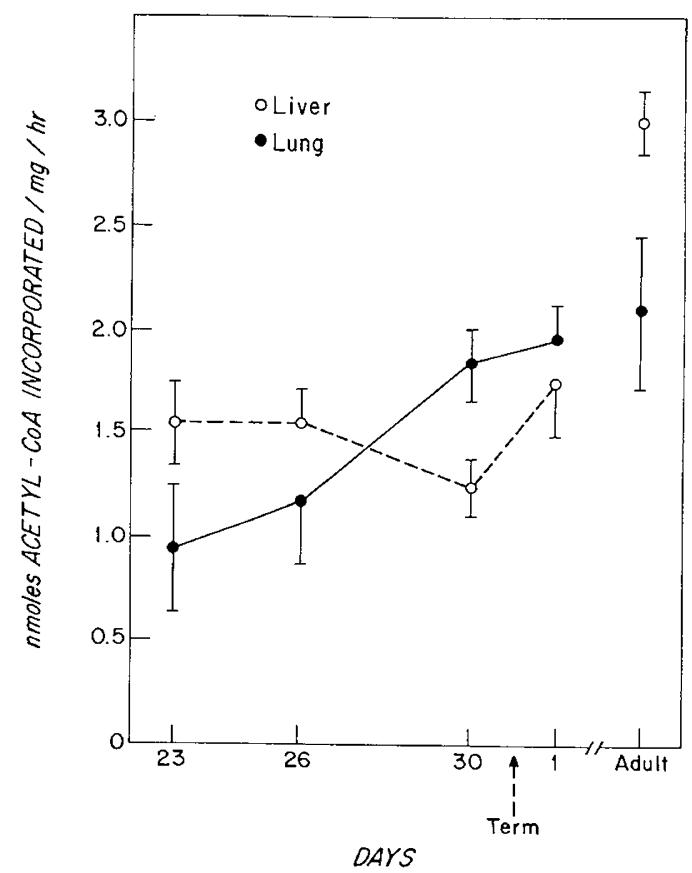

Fig. 4. Developmental profile of pulmonary and hepatic mitochondrial fatty acid elongation. Assay conditions are as described in the text. 
r:coverable radioactivity will be reduced proportionately. As is shown in Table I, 12.4\% of the label was recovered in the case of the pulmonary fatty acid synthetase system. This indicates de novo synthesis. Values of $88 \%$ and $58.7 \%$ were obtained from microsomal and mitochondrial synthesis, respectively. These latter values are consistent with chain elongation.

Gas-liquid chromatography of the fatty acids synthesized by the supernatant pathway revealed that the main product was palmitic acid (Fig. 5), as has been shown to be the case with liver [3]. Figure 6 shows the products of pulmonary microsomal fatty acid synthesis. Palmityl-CoA was added to the assay medium as a substrate primer. The major products were stearic (18:0), 20-carbon unsaturated, and 22-carbon unsaturated fatty acids. This reflects chain elongation of the 16-carbon primer and probably also of endogenous pulmonary fatty acids. The products of pulmonary mitochondrial fatty acid synthesis are shown in Figure 7. Palmitylcarnitine was added as a substrate primer for mitochondrial elongation. The results are similar to those obtained with the microsomal system with stearic acid

Table I. Decarboxylation of pulmonary fatty acids

\begin{tabular}{lc} 
Pathway & $\begin{array}{c}\text { Radioactivity in carboxyl } \\
\text { carbon, } \%\end{array}$ \\
\hline Supernatant & 12.4 \\
Microsomal & 88.0 \\
Mitochondrial & 58.7 \\
\hline
\end{tabular}

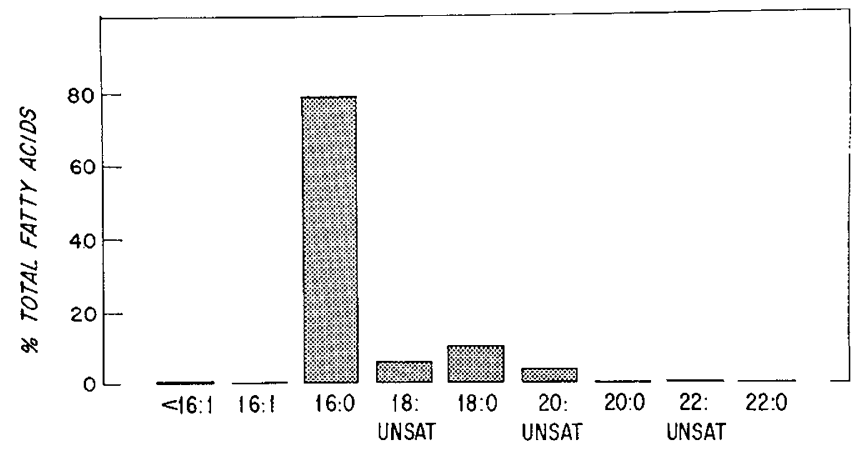

Fig. 5. Products of pulmonary fatty acid synthetase. Gas chromatographic analysis of the methyl ester of fatty acids synthesized by pulmonary supernatant with $\left(1,3-{ }^{14} \mathrm{C}\right)$ malonyl-CoA as substrate. The radioactivity incorporated into each fatty acid has been measured. The percentage of total fatty acids on the ordinate refers to the percentage of total recoverable radioactivity found in each fatty acid fraction. Conditions of assay and analysis as described in the text. Identification was achieved by simultaneous chromatography of standard methyl esters of fatty acids. UNSAT: unsaturated.

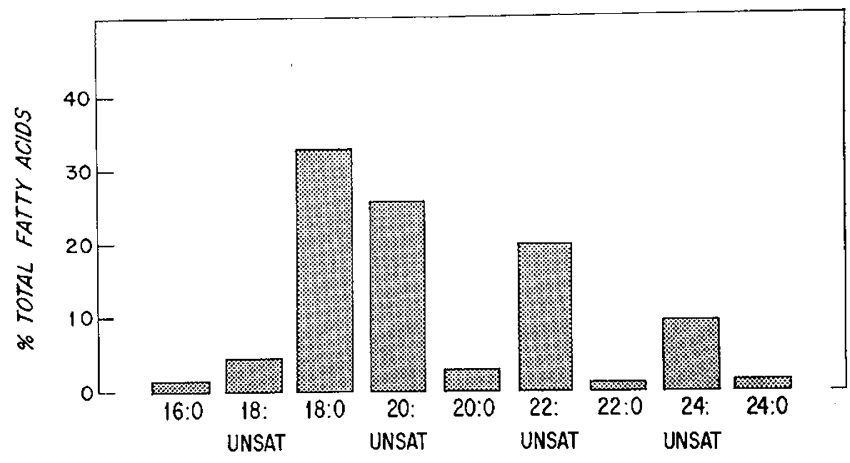

Fig. 6. Products of pulmonary microsomal fatty acid synthesis. Gas chromatographic analysis of the methyl ester of fatty acids synthesized by the pulmonary microsomal fraction with ( 1,3 $\left.{ }^{14} \mathrm{C}\right)$ malonyl-CoA as substrate. Conditions as described in text. Palmityl-CoA was added as primer. UNSAT: unsaturated.

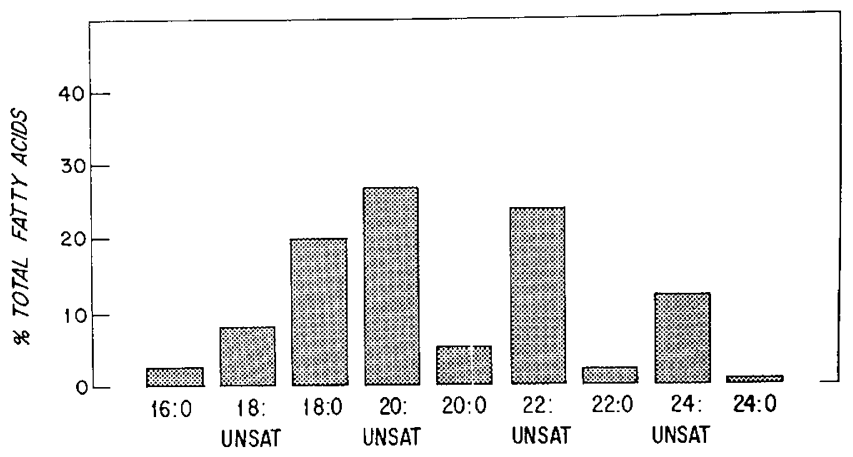

Fig. 7. Products of puImonary mitochondrial fatty acid synthesis. Gas chromatographic analysis of the methyl ester of fatty acids synthesized by the pulmonary mitochondrial fraction with $\left(\mathrm{l}-{ }^{14} \mathrm{C}\right)$ acetyl-CoA as substrate. Conditions as described in text. Palmitylcarnitine was added as primer. UNSAT: unsaturated.

and 20- and 22-carbon unsaturated fatty acids predominating.

\section{Discussion}

It is generally recognized that fatty acid synthesis can be carried out by at least three subcellular fractions. De novo synthesis from acetyl-CoA occurs in the soluble supernatant fraction of the cell as indicated in the following sequence.

$$
\text { Acetyl-CoA }+\mathrm{CO}_{2} \rightarrow \text { malonyl-CoA }
$$

Acetyl-CoA +7 malonyl-CoA $\rightarrow$ palmitic acid

The initial formation of malonyl-CoA is catalyzed by acetyl-CoA carboxylase which is thought to be the ratelimiting step in de novo synthesis [15]. Synthesis of palmitic acid is then carried out by the fatty acid synthetase complex [3]. Chain elongation of fatty acids in the mitochondrial $[11,23]$ and the microsomal [16] frac 
tions of the cell involves elongation of preexisting fatty acids by 2 carbon units. In the mitochondrial system, the 2-carbon source is acetyl-CoA and in the microsomal system the carbons are derived from malonylCoA.

Palmitic acid, the main product of de novo synthesis, is a major component of dipalmityl lecithin, an important surface active lipid. Our data indicate that fatty acids can be synthesized in the fetal rabbit lung by the de novo pathway and to a lesser extent by chain elongation. Not only is the fetal lung capable of de novo synthesis, but fatty acid synthetase activity is high early in fetal life, well before the lung is functionally mature and able to produce adequate surface active lipids (29 days). Whereas pulmonary fatty acid synthetase activity is fairly constant in the fetus and during the first days of life, hepatic activity reaches very high levels during fetal life but drops markedly at birth. Ballard and Hanson [1] showed that hepatic lipogenesis from glucose and acetate is influenced by diet. Activity is depressed during the suckling period when fatty acid needs are met by the high fat milk diet, and rises again after weaning. Similar developmental patterns for citrate lyase, acetyl-CoA synthetase, acetyl-CoA carboxylase, and fatty acid synthetase have recently been reported by Illife et al. [12].

It is interesting that pulmonary fatty acid synthetase and acetyl-CoA carboxylase activity appears to be unaffected by birth and nutritional influences. Felts [6], in experiments with adult rat lung, found that fasting delays the esterification of fatty acid into triglycerides but that esterification into phospholipids was independent of nutritional status [17]. Faridy [5], however, has shown that whereas water deprivation increases lung lecithin content in rats, food deprivation results in lowered lung lecithin content and an increased tendency for the excised lungs to collapse. Thus, the data on the effect of diet on lung lipid synthesis is somewhat conflicting at the present time and warrants further study.

Pulmonary and hepatic fatty acid elongation do not appear to be the major routes of fatty acid synthesis in the fetal rabbit. Microsomal elongation activity is low in the fetal and early newborn period, adult activity being fourfold greater. In the newborn rat, it has been shown [2l] that there is an increase in hepatic microsomal elongation activity after birth, probably associated with development of the membrane components of hepatic endoplasmic reticulum. This is followed by a fall in activity after 13 days of age which persists until the time of weaning when there is again an increase, which indicates that this pathway, too, is influenced by diet.

Pulmonary mitochondrial elongation becomes active in late fetal life, but quantitatively appears less important than de novo synthesis. This data is at variance with that of Tombropolous [19], who reported that mitochondrial fatty acid synthesis was the most active pathway in lung. The hepatic pattern of relatively low prenatal activity is similar to that described previously [22] in developing rat heart. Myocardial fatty acid elongation in the rat is very low before birth, but increases rapidly during the first week of life.

The pulmonary mitochondrial pathway produced a greater percentage of fatty acids with chain length above 18 carbons than did the microsomal pathway. This probably represents two stage elongation and may account for the lower recovery of radioactivity after decarboxylation of the products of the mitochondrial system.

Our results support the finding of Schiller and Bensch [17] that the enzymes for fatty acid synthesis are active in rabbit lung and show that fatty acid synthetase is active early in fetal development. This ensures a source of fatty acid for lung membrane growth and for surfactant formation when the pathways for lecithin synthesis mature.

\section{Summary}

Fetal rabbit lung synthesizes fatty acids de novo and by chain elongation. The enzymes of the de novo pathway are the major ones and mature early; their activity is fairly constant from 23 days of gestational age onwards. This developmental pattern is markedly different from that of the enzymes of hepatic de novo fatty acid synthesis, which are very active during fetal life but decrease in activity with birth and possibly the associated ingestion of a high fat milk diet. Both pulmonary and hepatic microsomal fatty acid elongation are relatively inactive during the fetal and early newborn period. Pulmonary mitochondrial elongation activity increases progressively during fetal life so that adult values are attained by the time of birth, but quantitatively this pathway appears less important than de novo synthesis.

The major product of pulmonary fatty acid synthetase is palmitic acid, whereas both the mitochondrial and microsomal elongation pathways produce mainly stearic acid and 20- and 22-carbon unsaturated fatty acids. 


\section{References and Notes}

1. Ballard, F. J., and Hanson, R. W.: Changes in lipid synthesis in rat liver during development. Biochem. J., 102: 952 (1967).

2. Brady, R. O., Bradley, R. M., and Trams, E. G.: Biosynthesis of fatty acids. J. Biol. Chem., 235: 3093 (1960).

3. BRESSLER, R., AND WAKIL, S. J.: Studies on the mechanism of fatty acid synthesis. IX. The conversion of malonyl coenzyme A to long chain fatty acids. J. Biol. Chem., 236: 1643 (1961).

4. CHIDA, N., AND ADAMs, F. H.: Incorporation of acetate into fatty acids and lecithin by lung slices from fetal and newborn lambs. J. Lipid Res., 8: 335 (1967).

5. FARIDY, E. E.: Effect of food and water deprivation on surface activity of lungs of rats. J. Appl. Phys., 29: 493 (1970).

6. Felts, J. M.: Biochemistry of the lung. Health Phys., 10:973 (1964).

7. Gluck, L., Landowne, R. A., and Kulovich, M. V.: The biochemical development of surface activity in mammalian lung. III. Structural changes in lung lecithin during development of the rabbit fetus and newborn. Pediat. Res., 4: 352 (1970).

8. Gluck, L., Motoyama, E. K., Smits, H. L., And Kulovich, M. V.: The biochemical development of surface activity in mammalian lung. I. The surface active phospholipids, the separation and distribution of surface active lecithin in the lung of the developing rabbit fetus. Pediat. Res., 1: 237 (1967).

9. Gluck, L., Scribney, M., and Kulovich, M. V.: The biochemical development of surface activity in mammalian Iung. II. The biosynthesis of phospholipids in the lung of the developing rabbit fetus and newborn. Pediat. Res., 1: 247 (1967).

10. Harlan, W. R., Said, S. I., Spuers, C. L., Banerjee, C. M., and Avery, M. E.: Synthesis of pulmonary phospholipids. Clin. Res., 12: 291 (1964).

11. Harlan, W. R., JR., AND WAKIL, S. J.: Synthesis of fatty acids in animal tissues. I. Incorporation of ${ }^{14} \mathrm{C}$-acetyl coenzyme $\mathrm{A}$ into a variety of long chain fatty acids by subcellular particles. J. Biol. Chem., 238: 3216 (1963).

12. Illife, J., KNight, B. L., AND Myant, N. B.: Fatty acid synthesis in the brown fat and liver of foetal and newborn rabbits. Biochem. J., 134: 341 (1973).

13. Jacobs, E. E., Jacobs, M., SAnadi, D. R., and Bradley, L.: Uncoupling of oxidative phosphorylation by the cadmium ion. J. Biol. Chem., 223: 147 (1956).

14. Klalis, M. H., Clements, J. A., and Havel, R. J.: Composition of surface active material isolated from beef lung. Proc. Nat. Acad. Sci. U. S. A., 47: 1858 (1961).
15. Martin, D. B., AND Vagelos, P. R.: Control by the tricarboxylic acid cycle of fatty acid synthesis. J. Biol. Chem., 237: 1787 (1962).

16. Nugteren, D. H.: The enzymic chain elongation of fatty acids by rat liver" microsomes. Biochim. Biophys. Acta, 106: 280 (1965).

17. SCHILler, H., AND Bensch, K.: De novo fatty acid synthesis and elongation of fatty acids by subcellular fractions of lung. J. Lipid. Res., 12: 248 (1971).

18. Stoffel, W., Chu, F., ANd Ahrens, E. H., JR.: Analysis of long chain fatty acids by gas-liquid chromatography. Micro-method for preparation of methyl esters. Anal. Chem., 31: 307 (1959).

19. Tombropoulos, E. G.: Fatty acid synthesis by subcellular fractions of lung tissue. Science, 146: 1180 (1964).

20. WARshaw, J. B.: Cellular energy metabolism during fetal development. IV. Fatty acid activation, acyl transfer and fatty acid oxidation during development of the chick and rat. Develop. Biol., 28: 537 (1972).

21. WARSHAW, J. B., AND KIMURA, R. E.: Changes in hepatic microsomal fatty acid synthesis during development of the rat. Biol. Neonate, 22: 133 (1973).

22. Warshaw, J. B., ANd Kimura, R. E.: Cellular energy metabolism during fetal development. V. Fatty acid synthesis by the developing heart. Develop. Biol., 33: 224 (1973).

23. Wrt-Peeters, E. M.: Synthesis of long-chain fatty acids in mitochondria. Biochim. Biophys. Acta, 176: 453 (1969).

24. Wayne, N. J.

25. Brinkman Instruments, Westbury, N. Y.

26. Beckman Instruments, Inc., Palo Alto, Calif.

27. Varian, Palo Alto, Calif.

28. Boston, Mass.

29. St. Louis, Mo.

30. Osaka, Japan.

31. The authors are grateful for the technical assistance of Mrs. Dana Frederick and Mrs. Helen Jones.

32. Dr. I. Gross was supported by a James Hudson Brown Fellowship from Yale University School of Medicine.

33. This research was supported by United States Public Health Service Grant no. HD-8293 and Connecticut Heart Association Grant no. 536.

34. Requests for reprints should be addressed to: J. B. WARSHAW, M.D., Department of Pediatrics, Yale University School of Medicine, 333 Cedar St., New Haven, Conn. 06510 (USA).

35. Accepted for publication October 30, 1973. 